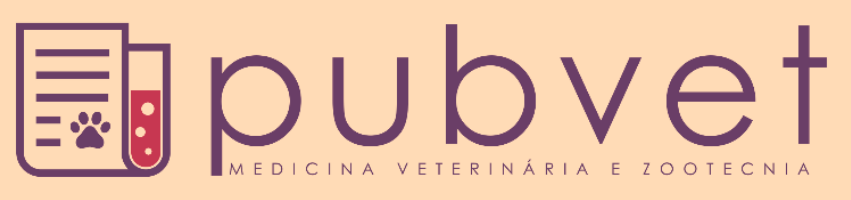

https://doi.org/10.31533/pubvet.v14n3a521.1-6

\title{
Inclusão de soro de leite na dieta de leitões na fase pré-desmama e creche
}

\author{
Emanuel de Souza Oliveira ${ }^{1^{*} \bullet}$, Allan Cerqueira de Oliveira $^{1 \bullet}$, Roberto Sobral Félix $^{1 \bullet}$, Daniel \\ Pereira Franklin Neto $^{10}$, Ronaldo Santos de Jesus ${ }^{10}$, Guilherme Rodrigues de Nascimento ${ }^{20}$ \\ ${ }^{1}$ Discente do curso de bacharelado em Zootecnia- Instituto Federal de Educação, Ciências e Tecnologia Baiano, Campus Santa Inês - \\ Bahia, Brasil. \\ ${ }^{2}$ Professor no Instituto Federal de Educação, Ciências e Tecnologia Baiano, Campus Santa Inês - Bahia, Brasil. \\ *Autor para correspondência, E-mail: emanuel_123-souza@hotmail.com
}

Resumo. O objetivo do experimento foi avaliar o desempenho e avaliação econômica da utilização do soro de leite na dieta de leitões no período de creche. O estudo aconteceu no setor de suinocultura do Instituto Federal Baiano - Campus Santa Inês, utilizando 16 leitões Large White, dividido em duas fases, sendo a primeira na maternidade com dois tratamentos no período pré-desmame, como período pré-experimental, ofertando soro de leite com ração na proporção de 50/50 para uma leitegada (T1) e para a outra sem o fornecimento de soro, apenas aleitamento natural (T2) com duração de sete dias antes do desmame. Na segunda fase, fase experimental, os leitões foram alojados e distribuídos em quatro tratamentos na creche seguindo o método de delineamento em blocos casualizados (DBC), contabilizando quatro tratamentos com duas repetições. As leitegadas do período pré-experimental foram distribuídas em quatro baias onde foram subdivididas em dois tratamentos, um com oferta do soro de leite com a ração, dieta úmida, na proporção de $30 \%$ de soro e $70 \%$ de ração formando duas repetições com soro e duas só com tratamento controle com fornecimento de ração seca. Os dados obtidos foram submetidos à análise de variância e interação $2 \times 4$, sendo as diferenças significativas entre as médias comparadas pelo teste de Tukey a $5 \%$ de probabilidade. Segundo a análise estatística houve diferença entre os tratamentos para os parâmetros de ganho de peso médio (GPM) e conversão alimentar (CA), sendo que os melhores resultado para esses parâmetros foram para os leitões alimentados com ração, já para o consumo de ração (CR) não foi observado diferença entre os tratamentos. A avaliação econômica se mostrou viável, ou seja, a pesar de não ter maximizado os resultados de desempenho, a inclusão de $30 \%$ do soro de leite na dieta de leitões na fase de creche pode ser uma ótima alternativa para economizar com custo da ração e dar um opção adequado de descarte desse subproduto para os laticínios.

Palavras chave: desempenho, avaliação, consumo, tratamentos

\section{Inclusion of whey in the diet of pre-weaning and nursery piglets}

Abstract. The objective of the experiment was to evaluate the performance and economic evaluation of the use of whey in the piglet diet during nursery. The study took place in the swine sector of the Federal Institute of Bahia-Campus Santa Inês using 16 Large White piglets. It was divided into two phases, the first in the maternity with two treatments in the period pre-weaning. In this period was offered whey with feed (ration) a proportion of $50 / 50$ for one litter (T1) and for the other treatment only natural breastfeeding (T2), without whey with duration of one week before weaning. In the second phase, experimental phase, the piglets were housed and distributed in four treatments in the nursery following the randomized block design (DBC) method, through four treatments with two repetitions each. The first phase T1 litter was distributed in four stalls where the whey was offered 
with $30 \%$ whey and $70 \%$ ration feed forming two repetitions with whey (ST1R1 and ST1R2) and two only with feed (RT1R1 and RT1R2). The first phase T2 litter was also distributed in four stalls with the same methodology followed for T1, with two repetitions adding 30\% whey in the diet (ST2R1 and ST2R2) and two only with feed (RT2R1 and RT2R2). The data obtained were submitted to analysis of variance and $4 \times 2$ interactions, with significant differences between the means compared by Tukey test at $5 \%$ probability. According to the statistical analysis, there was a difference $(\mathrm{P}<0.05)$ between treatments for the parameters of mean weight gain (GPM) and feed conversion (CA), numerically the best results for these parameters were for piglets fed only with feed, whereas for feed intake $(\mathrm{CR})$ no difference $(\mathrm{P}>0.05)$ was observed between treatments. The economic evaluation proved to be viable, that is, despite not maximizing the performance results, the inclusion of $30 \%$ whey in the nursery phase piglet diet can be a great alternative to save on feed cost and give an appropriate disposal option for this by-product to dairy products.

Keywords: performance, evaluation, consumption, treatments

\section{Inclusión de suero en la dieta de lechones pre-destete y de cría}

Resumen. El objetivo del experimento fue evaluar el rendimiento y la evaluación económica del uso de suero en la dieta de los lechones durante la cría. El estudio se realizó en el sector porcino del Instituto Federal de Bahía-Campus Santa Inês utilizando 16 lechones Large White, dividido en dos fases, la primera en la maternidad con dos tratamientos en el período previo al destete, como período preexperimental, ofreciendo suero y concentrado en la proporción 50/50 para una camada (T1) y el otro grupo sin el suministro de suero de leche, solo lactancia materna (T2) con duración de siete días antes del destete. En la segunda fase, fase experimental, los lechones fueron distribuidos en 4 tratamientos y alojados en el corral siguiendo el método de diseño de bloques al azar (DBC), lo que representa cuatro tratamientos con dos repeticiones cada uno. La camada T1 de la primera fase se distribuyó en cuatro espacios donde se ofreció el $30 \%$ de suero y $70 \%$ de ración, ofreciendo dos repeticiones con suero (ST1R1 y ST1R2) y dos con solo ración. (RT1R1 y RT1R2). La camada de T2 de la primera fase también se distribuyó en cuatro espacios con la misma metodología seguida para $\mathrm{T} 1$, con dos repeticiones que añadieron $30 \%$ de suero en la dieta (ST2R1 y ST2R2) y dos con ración solamente (RT2R1 y RT2R2). Los datos obtenidos se sometieron a análisis de varianza e interacción 4x2, con diferencias significativas entre las medias en comparación con la prueba de Tukey con una probabilidad del 5\%. Según el análisis estadístico, hubo diferencia entre los tratamientos para los parámetros de peso promedio y de conversión alimenticia, siendo los mejores resultados para los lechones alimentados únicamente con ración. Sin embargo, para el consumo de alimento no se observó diferencia entre tratamientos. La evaluación económica demostró ser viable, es decir, a pesar de no maximizar los resultados de rendimiento, la inclusión del 30\% de suero en la dieta de lechones en la fase de cría puede ser una gran alternativa para ahorrar en el costo de los alimentos y dar una opción de eliminación adecuada para este subproducto a los lacticinios.

Palabras clave: rendimiento, evaluación, consumo, tratamientos

\section{Introdução}

A suinocultura vem crescendo muito nos últimos anos tendo um aumento de $42,7 \%$ na produção mundial de carne, onde em 1995 eram produzidos 78,2 milhões de toneladas e em 2012 passou a produzir 111,7 milhões de toneladas (ANUALPEC, 2019). Enquanto a produção de carne cresceu $42,7 \%$, o crescimento do plantel foi apenas $7,1 \%$, isso pode ser explicado pelos avanços na área da genética e da nutrição fazendo com que os animais cheguem ao abate mais precocemente (Roppa, 2014).

A fase de creche é o período da criação de suínos que vai desde o desmame até cerca de 65 dias de vida, onde os leitões podem chegar a pesar $20 \mathrm{~kg}$ de peso. Contudo, nas criações comerciais, o desmame 
é precoce e por isso é necessário um maior cuidado com o manejo, nutrição e sanidade (Campos et al., $\underline{2010)}$.

Quadros et al. (2002) explicam que a mudança de alimentação no momento do desmame leva os leitões a passar por uma adaptação repentina para poder digerir o amido e o óleo vegetal em vez de lactose e gordura do leite, influenciando nos resultados de ganho de peso, consumo e conversão alimentar.

A utilização de ingredientes lácteos nas rações pré-iniciais é comum; pois proporcionam melhor desempenho dos leitões visto que contribuem com o ambiente estomacal para melhorar absorção dos nutrientes (Silva et al., 2008). Um dos derivados do leite que possui potencial nutricional é o soro de leite que apresenta alta quantidade proteica relacionada à boa palatabilidade, digestibilidade e presença de hemoglobinas. Ainda também contribui com a redução do $\mathrm{pH}$ estomacal dos leitões que é importante para a produção de enzimas digestivas (Kummer et al., 2009). Outro fato importante como alternativas de utilização deste subproduto é que devido à presença de substâncias orgânicas o soro de leite se torna um potencial poluente, pois possui uma baixa biodegradabilidade (Prazeres et al., 2012).

O objetivo deste trabalho foi avaliar o desempenho e avaliação econômica da utilização de soro de leite na dieta de leitões no período de creche.

\section{Material e métodos}

O experimento foi realizado no Setor de Suinocultura do Instituto Federal de Educação, Ciências e Tecnologia Baiano, Campus Santa Inês, situado na BR - 420 entre Ubaíra e Santa Inês - Ba. Foram utilizados 16 leitões Large White, dividido em duas fases, sendo a primeira na maternidade com dois tratamentos no período pré-desmame, como período pré-experimental, ofertando soro de leite com ração na proporção de 50/50 para uma leitegada (T1) e para a outra sem o fornecimento de soro, apenas aleitamento natural (T2) com duração de sete dias antes do desmame.

Na segunda fase houve o alojamento e distribuição dos leitões em oito baias na creche seguindo o método de delineamento de blocos casualizados (DBC), ficando quatro tratamentos com duas repetições cada. A leitegada do T1 da primeira fase foi distribuída em quatro baias com a oferta de soro de leite com a ração na proporção de $30 \%$ de soro e $70 \%$ de ração formando duas repetições com soro (ST1R1 e ST1R2) e duas só com ração (RT1R1 e RT1R2). A leitegada do T2 da primeira fase também foi distribuída em quatro baias com a mesma metodologia seguida para o $\mathrm{T} 1$, formando duas repetições com soro e ração (ST2R1 e ST2R2) e duas só com ração (RT2R1 e RT2R2).

Utilizou-se durante todo o período experimental ração inicial industrial da Master Alimentos LTDA (Leitão do Sítio Master $40 \mathrm{~kg}$ ).

Parâmetros avaliados: consumo de ração em $\mathrm{kg}(\mathrm{CR})$, conversão alimentar (CA), ganho de peso médio em kg (GPM) e viabilidade econômica (VE). Os dados de ganho médio diário de peso foram obtidos por pesagens individuais desde o início do experimento semanalmente até os 35 dias pósdesmame (cinco semanas); os dados referentes ao consumo diário de ração foram obtidos pela quantidade de ração fornecida medida diariamente e, totalizando e subtraindo dos restos presente nos comedouros no final de cada período.

A viabilidade econômica da utilização do soro de leite na dieta de leitões na fase de creche foi estudada pelo modelo proposto por Bertol et al. (1996): RE x PR $=(\mathrm{CUT} \times \mathrm{Km} \times 2 / \mathrm{N}) \times \mathrm{K}$. Onde RE = ração economizada durante o período de utilização do soro; $\mathrm{PR}=$ preço da ração; $\mathrm{CUT}=$ custo do quilômetro rodado pelo veículo a ser utilizado; $\mathrm{Km}$ = distância da granja ao laticínio; $\mathrm{K}=$ número de viagens efetuadas durante o período de utilização do soro (multiplicado por dois, sendo uma ida e uma volta); $\mathrm{N}=$ número de animais alimentados.

O custo de transporte por quilômetro rodado em um Chevrolet Prisma ano 2019 é de R\$ 0,306, incluindo todos os custos fixos e variáveis. O custo da ração inicial sendo de $\mathrm{R} \$ 1,51 / \mathrm{kg}$, a distância entre o IF Baiano e o laticínio de $17 \mathrm{~km}$, transporte realizado em dois dias e fornecimento a 8 animais (com $30 \%$ de substituição da ração por soro). 
Os dados obtidos foram submetidos à análise de variância e interação $4 \times 2$, sendo as diferenças significativas entre as médias comparadas pelo teste de Tukey a 5\% de probabilidade.

\section{Resultados e discussão}

Os resultados de desempenho dos leitões referente ao ganho de peso médio (GPM), consumo de ração (CR) e conversão alimentar (CA) na fase de creche estão representados na tabela 1 . O consumo de ração não apresentou diferença $(P>0,05)$ entre os tratamentos, ou seja, estatisticamente os leitões tiveram consumo semelhante de ração independentemente da dieta que foram submetidos neste período.

Quanto ao ganho de peso médio houve diferença $(\mathrm{P}<0,05)$ entre os tratamentos. Quando comparada as médias pelo teste de Tukey com grau de confiança de $95 \%$, as médias do tratamento 1 (soro/ração no prétratamento e com ração na creche), tratamento 2 (soro/ração no pré-tratamento e com soro/ração na creche), tratamento 3 (sem soro/ração no pré-tratamento e com ração na creche) estatisticamente são semelhantes, o tratamento 2 e tratamento 4 (sem soro/ração no pré-tratamento e com soro/ração na creche) estatisticamente também não apresentam diferença significativa $(>0,05)$ entre as médias. $\mathrm{O}$ tratamento 4 obteve média inferior quando comparada ao tratamento 1 e 3 que tiveram oferta apenas de ração seca na creche. Numericamente o tratamento 1 que teve oferta de ração com soro de leite na proporção de 50/50 no préexperimental e ração seca na fase experimental (RT1) teve a melhor média. Segundo Bertol et al. (1996), o soro de leite apresenta baixo teor de matéria seca (MS), ficando em torno de 5,0\% a 7,0\%, isso causou um desbalanceamento de nutrientes na dieta quando substituído a fração de $30 \%$ da ração fornecida pelo soro de leite.

Contudo, o tratamento 2 que teve soro de leite na dieta no período experimental e no préexperimental (ST1) estatisticamente obteve a média constante comparando-a com as do tratamento $1 \mathrm{e}$ 3, ou seja, ajustando os níveis na dieta este subproduto pode ser utilizado na alimentação de leitões na fase de creche em vez de ser descartados no meio ambiente. Ainda segundo Bertol et al. (1996), leitões pós-desmamados podem ter um fornecimento de $20 \%$ de substituição da ração por soro de leite na dieta.

Os dados de conversão alimentar mostram que houve diferença $(P<0,05)$ entre os tratamentos e ao comparar as médias pelo teste de Tukey com grau de confiança de $95 \%$, o tratamento 1,2 e 3 não apresentam diferença significativa entre as médias e o tratamento 2 e 4 também. Isso implica dizer, que a substituição de $30 \%$ de soro de leite na dieta com ração inicial não melhora a conversão alimentar e também não vai haver piora, já de acordo com Hauptli et al. (2005), a inclusão de $21 \%$ de soro de leite na dieta de leitões na fase de creche melhora a conversão alimentar.

Tabela 1. Resultados de desempenho dos leitões no período de creche após o desmame aos 28 dias e suplementados com soro de leite.

\begin{tabular}{|c|c|c|c|c|c|c|}
\hline \multirow{4}{*}{$\begin{array}{l}\text { Pré-experiemental } \\
\text { Experimental }\end{array}$} & \multicolumn{4}{|c|}{ Tratamentos } & \multirow{4}{*}{ Valor-P } & \multirow{4}{*}{$\mathrm{CV}$} \\
\hline & \multicolumn{2}{|c|}{ T1 - Soro + ração } & \multicolumn{2}{|c|}{ T2 - ração } & & \\
\hline & 1 (RT1) & 2 (ST1) & 3 (RT2) & $4(\mathrm{ST} 2)$ & & \\
\hline & Ração seca & Soro + Ração & Ração seca & Soro + Ração & & \\
\hline Ganho de peso médio, kg & $5,530 \pm 0,0177 \mathrm{~A}$ & $4,760 \pm 0,212 \mathrm{AB}$ & $5,120 \pm 0,247 \mathrm{~A}$ & $3,914 \pm 0,316 \mathrm{~B}$ & 0,008 & 0,227 \\
\hline consumo de ração, $\mathrm{kg}$ & $14,560 \pm 1,49 \mathrm{~A}$ & $16,192 \pm 0,0283 \mathrm{~A}$ & $15,444 \pm 0,384 \mathrm{~A}$ & $15,943 \pm 0,283 \mathrm{~A}$ & 0,304 & 0,782 \\
\hline conversão alimentar & $2,632 \pm 0,261 \mathrm{~A}$ & $3,405 \pm 0,146 \mathrm{AB}$ & $3,022 \pm 0,221 \mathrm{~A}$ & $4,084 \pm 0,258 \mathrm{~B}$ & 0,012 & 0,226 \\
\hline
\end{tabular}

Seguindo a metodologia foi feito uma análise fatorial para ver se há interação entre o préexperimental e experimental (creche) nos resultados de ganho de peso médio, consumo de ração e conversão alimentar, ou seja, se a inclusão de soro de leite com ração a vontade neste período vai influenciar nos parâmetros de desempenho. Os resultados estão representados na tabela 2.

Tabela 2. Resultados de interação entre fatores pré-experimental e experimental de suínos suplementados com soro de leite.

\begin{tabular}{lcccc}
\hline & & Variáveis & \\
\hline Fatores & Ganho de peso médio & Consumo de ração & Conversão alimentar \\
Pré-experimental Experimental & $($ Valor-P/F) & (Valor-P/F) & $($ Valor-P/F) & $0,417<0,82$ \\
\hline
\end{tabular}


Para todas as variáveis, sendo elas: ganho de peso médio (GPM), consumo de ração (CR) e conversão alimentar (CA) não houve interação $(\mathrm{P}<\mathrm{Valor})$ entre os fatores para nenhum dos períodos experimentais. Com base nesses resultados, justifica-se, que a oferta de soro de leite na proporção de $50 \%$ de soro e $50 \%$ de ração inicial no período de sete dias antes do desmame não contribui com o desenvolvimento da capacidade de produzir enzimas digestivas para digerir os ingredientes da ração, pois segundo Trindade Neto et al. (2002), a dieta ofertada no desmame não conduz qualitativa e quantitativamente com a produção enzimática desses leitões nessa fase. Nas três primeiras semanas, do nascimento ao desmame, esses leitões tem o organismo fisiologicamente adaptados para digerir o leite materno (Pascoal \& Silva, 2005).

\section{Avaliação econômica}

A avaliação econômica foi calculada de acordo com o modelo matemático sugerido por Bertol et al. (1996), expresso na fórmula a seguir:

$$
R E \times P R=(C U T \times K m \times 2 / N) \times K
$$

$\mathrm{RE}=$ ração economizada durante o período de utilização do soro; $\mathrm{PR}=$ preço da ração $/ \mathrm{kg} ; \mathrm{CUT}=$ custo do quilômetro rodado pelo veículo a ser utilizado; $\mathrm{Km}$ = distância da granja ao laticínio; $\mathrm{K}$

= número de viagens efetuadas durante o período de utilização do soro (multiplicado por dois, sendo uma ida e uma volta); $\mathrm{N}$ = número de animais alimentados.

Os resultados do estudo de avaliação econômica da substituição de $30 \%$ de ração pelo soro de leite em dietas para leitões na creche estão apresentados na tabela 3.

Tabela 3. Resultado da avaliação econômica da utilização de 30\% de soro de leite na dieta de leitões na fase de creche.

\begin{tabular}{lccc}
\hline & & Variáveis \\
\hline Animais $\left(\mathrm{n}^{\circ}\right)$ & Proporção da utilização de soro & Custo total da ração economizada por & Custo total do transporte por \\
08 & de leite na dieta $(\%)$ & leitão $(\mathrm{R} \$)$ & leitão $(\mathrm{R} \$)$ \\
\hline
\end{tabular}

O custo total da ração economizada foi maior que o custo total do transporte do soro, ou seja, houve uma redução de custo com a utilização $30 \%$ de soro de leite na dieta de leitões no período de creche. De acordo com Hauptli et al. (2005), os níveis de soro utilizado na dieta, assim como, a quantidade de animais, o número de viagens e a distância do laticínio vão influenciar nos resultados desses parâmetros de avaliação econômica.

O resultado do custo total do transporte por animal foi relativamente baixo porque o número de viagem foi reduzido levando em consideração a distância até o laticínio e o armazenamento deste subproduto em uma temperatura em torno de $5^{\circ} \mathrm{C}$ que de acordo com Bertol et al. (1996), mantem as características físicas, químicas e sensoriais do soro de leite por um grande período de tempo. No experimento foi utilizado 16 leitões dividido em 4 tratamentos com 2 repetições cada contabilizando 8 leitões alimentados com soro de leite, segundo Hauptli et al. (2005), quanto maior for o número de animais e a porcentagem de soro utilizado na dieta, menor vai ser o custo total da ração por leitão.

Conforme Moreira et al. (2000), o soro de leite é pouco aproveitado pelas indústrias de alimentos e apesar de ser aproveitado por alguns produtores de suínos como alimento alternativo, ainda se tem muitas dúvidas sobre a sua qualidade nutricional. Todavia, vem sendo muito estudada a utilização deste subproduto para dieta de suínos em diferentes fases de produção obtendo-se vários resultados satisfatórios com diferentes níveis de inclusão.

\section{Conclusão}

A utilização do soro de leite com inclusão de $30 \%$ na dieta de leitões em fase de creche não aumentou a média de ganho de peso em relação aos só alimentados com ração. O consumo de ração numericamente teve uma maior média para os que tiveram oferta do soro de leite, mas estatisticamente as médias foram iguais. Para o parâmetro de conversão alimentar os animais alimentados com ração tiveram melhores resultados. Na avaliação econômica da utilização do soro de leite o custo da ração economizada foi 
maior do que o custo do transporte do soro de leite se tornando viável economicamente podendo ser uma ótima alternativa para economizar com custo da ração e dar uma opção adequada de descarte desse subproduto para os laticínios.

\section{Referências bibliográficas}

ANUALPEC. (2019). Anuário da Pecuária Brasileira (20th ed., Vol. 1). Instituto FNP.

Bertol, T. M., Santos Filho, J. I., \& Bonett, L. (1996). Soro de leite integral na alimentação dos suínos. Suinocultura Dinâmica, 5(17), 1-8.

Campos, J. A., Tinôco, I. F. F., Silva, F. F., Pupa, J. M. R., \& Silva, I. J. O. (2010). Enriquecimento ambiental para leitões na fase de creche advindos de desmame aos 21 e 28 dias. Revista Brasileira de Ciências Agrárias, 5(2), 272-278.

Hauptli, L., Lovatto, P. A., Silva, J. H. S., Garcia, G. G., Brum Júnior, B., de Souza, B., \& Oliveira, J. L. S. de. (2005). Níveis de soro de leite integral na dieta de leitões na creche. Ciencia Rural, 35(5), 1161-1165.

Kummer, R., Gonçalves, M. A. D., Lippke, R. T., Marques, B. M. F., \& Mores, T. J. (2009). Fatores que influenciam o desempenho dos leitões na fase de creche. Acta Scientiae Veterinariae, 37(1), 195-209.

Moreira, A., Silva, A., \& Antunes, M. (2000). Soro de leite: de resíduo a alimento. Alimentos e Nutrição, 4, 32-35.

Pascoal, L. A. F., \& Silva, L. da P. G. (2005). Adição de enzimas exógenas nas dietas de leitões desmamados. Revista Eletrônica Nutritime, 2(6), 273-283.

Prazeres, A. R., Carvalho, F., \& Rivas, J. (2012). Cheese whey management: A review. Journal of Environmental Management, 110, 48-68.

Quadros, A. R. B., Kiefer, C., Henn, J. D., Scariot, G., \& Silva, J. H. S. (2002). Dietas simples e complexa sobre o desempenho de leitões na fase de creche. Ciência Rural, 32(1), 109-114.

Roppa, L. (2014). Panorama da Produção de Suínos no Brasil e no Mundo. In: Ferreira, A. H. et al. Produção de Suínos: Teoria e Prática. Brasília: Associação Brasileira dos Criadores de Suínos, cap. 1, p. 23-48.

Silva, A. M. R. da, Berto, D. A., Lima, G. J. M. M. de, Wechsler, F. S., Padilha, P. M., \& Castro, V. S. (2008). Valor nutricional e viabilidade econômica de rações suplementadas com maltodextrina e acidificante para leitões desmamados. Revista Brasileira de Zootecnia, 37(2), 286-295.

Trindade Neto, M. A., Barbosa, H. P., Petelincar, I. M., \& Schammass, E. A. (2002). Dietas para leitões nas fases de creche e diferentes idades ao desmame. Revista Brasileira de Zootecnia, 31(2), 687695.

Recebido: 8 de outubro, 2019.

Aprovado: 18 de novembro, 2019.

Publicado: 22 de abril, 2020.

Licenciamento: Este artigo é publicado na modalidade Acesso Aberto sob a licença Creative Commons Atribuição 4.0 (CC-BY 4.0), a qual permite uso irrestrito, distribuição, reprodução em qualquer meio, desde que o autor e a fonte sejam devidamente creditados. 